\title{
Augmentation of Perceived Sweetness in Sugar Reduced Cakes by Local Odor Display
}

\author{
Heikki Aisala $^{1}$, Jussi Rantala ${ }^{2}$, Saara Vanhatalo ${ }^{1}$, Markus Nikinmaa ${ }^{1}$, \\ Kyösti Pennanen ${ }^{1}$, Roope Raisamo ${ }^{2}$ and Nesli Sözer ${ }^{1}$ \\ ${ }^{1}$ VTT Technical Research Centre of Finland \\ Espoo, Finland \\ ${ }^{2}$ Faculty of Information Technology and Communication Sciences \\ TAUCHI Research Center, Tampere University, Finland
}

\begin{abstract}
Multisensory augmented reality systems have demonstrated the potential of olfactory cues in the augmentation of flavor perception. Earlier studies have mainly used commercially available sample products. In this study, custom rye-based cakes with reduced sugar content were used to study the influence of different odorants on the perceived sweetness. A custom olfactory display was developed for presenting the odorants. The results showed that augmentation of a reduced sugar rye-based cake with localized maltol, vanilla, and strawberry odor increased the perceived sweetness of the cake-odor pair compared to a cake with deodorized airflow.
\end{abstract}

\section{CCS CONCEPTS}

- Human-centered computing $\rightarrow$ Human computer interaction $\rightarrow$ Empirical studies in $\mathrm{HCI}$

- Human-centered computing $\rightarrow$ Human computer interaction $\rightarrow$ Interaction paradigms $\rightarrow$ Mixed / augmented reality

\section{KEYWORDS}

Perceived sweetness, human-food interaction, odors, odor display, flavor

\section{ACM Reference format:}

Heikki Aisala, Jussi Rantala, Saara Vanhatalo, Markus Nikinmaa, Kyösti Pennanen, Roope Raisamo and Nesli Sözer. 2020. Augmentation of Perceived Sweetness in Sugar Reduced Cakes by Local Odor Display. In Companion Publication of the 2020 International Conference on Multimodal Interaction (ICMI '20 Companion), October 25-29, 2020, Virtual event, Netherlands. ACM, New York, NY, USA, 6 pages. https://doi.org/10.1145/3395035.3425650

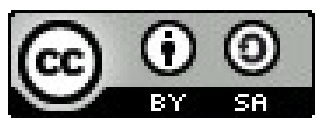

This work is licensed under a Creative Commons Attribution-ShareAlike International 4.0 License.

ICMI'20 Companion, October 25-29, 2020, Virtual Event, Netherlands.

(C) 2020 Copyright is held by the owner/author(s).

ACM ISBN 978-1-4503-8002-7/20/10.

https://doi.org/10.1145/3395035.3425650

\section{INTRODUCTION}

Today's modern consumer lives in a contradictory situation being surrounded by an abundance of delicious high-calorie foods but trying to maintain a healthy diet. In addition to healthiness, expectations towards the eating experience are high. For example, elements of pleasure, creativity, and playfulness are desired from the foods. The food should meet nutritional needs and hedonic needs to lead to 'reward homeostasis' which, in turn, is expected to decrease the risk of overeating [31]. Although healthy foods fulfill the homeostatic needs, the hedonic needs are not necessarily fulfilled. With new technology and Human-Food Interaction (HFI) [5], healthy foods could be augmented to enhance the eating experience in a customized fashion.

Because eating is a highly multisensory event where all the five senses contribute to the overall perception of flavor [8,25,32], HFI researchers have created visual $[8,21]$, auditory $[3,12,28,40]$, haptic $[7,9]$, olfactory [17-19,38], and gustatory [26,27] cues associated with the flavor. One particularly promising technology platform for flavor augmentation is augmented reality which enables superimposing digital information over real world views [6]. For example, the MetaCookie+ augmented reality system presented visual and olfactory cues for altering the flavor of a cookie $[19,20]$. Olfactory cues are an essential part of taste perception which largely originates from the sense of smell [2]. Olfactory cues can also enhance or suppress the perception of tastants and vice versa $[34,39]$. The results of an exploratory study with the MetaCookie+ system indicated that most participants experienced a change in the flavor with the added visual and olfactory cues [19]. Similar systems with visual and olfactory cues have also been developed for VR $[10,33,37]$ and a general olfactory toolkit OWidgets has been published [16] to facilitate this design.

While the earlier multisensory systems have demonstrated the potential of AR in augmenting flavor perceptions, they have utilized small-scale user evaluations [6,41]. Also, the process of selecting olfactory cues has not been in the focus of earlier work [19,37,38]. Our research project titled "Augmented Eating Experiences" aims to contribute to this line of research by studying how food products augmented with different sensory cues can modify eating experiences. The goal of our research is to understand whether augmented food products can assist in promoting health and well- 
being, for example, by modifying the perceived sweetness of food. A consumer could choose a low sugar, healthy product that is equivalent to a regular product in the sensory experience.

Eating experience, as defined here, is a complex phenomenon where individual's sensory evaluations, physiological cues (such as satiety), emotional responses to eating situation, mood, perceived vitality, satisfaction, food reward and psycho-physiological responses to food are crucial (e.g. [4,13,15,22-24,30]). This initial workshop paper focuses on sensory evaluations.

The aim of this study was to explore how olfactory cues can be used to augment the perceived sweetness of sugar reduced healthy cakes. The influence of various locally delivered odors on the perceived sweetness of reduced sugar healthy rye cakes was evaluated.

\section{METHODS}

\subsection{Participants}

The assessors were members of VTT's trained sensory panel. These assessors were selected by testing their senses of smell, taste, and color vision and trained extensively in the use of descriptive, quantitative sensory evaluation techniques and in the use of line scales to evaluate sensory intensities. The selection and training followed the standard protocols for analytical sensory panels $[11,14]$. There were 11 assessors in study 1 ( 3 males and 8 females, 30 to 58 years old, average age 44 years, standard deviation 10 years) and 10 assessors in study 2 (3 males and 7 females, 30 to 58 years old, average age 40 years, standard deviation 9 years). The assessors were trained to the specific evaluation protocol, the different samples, and the sensory attributes before the main evaluations in a consensus training session before each study.

An application regarding the sensory evaluation protocol was made to VTT's internal ethical committee whose recommendations were followed in the evaluation. All panelists provided a written informed consent before the evaluations and were aware that the study aimed to investigate how the eating experience can be modified with augmented reality technologies.

\subsection{Odors}

In the first phase, 12 sweet candidate odor compounds and commercial products were evaluated for odor congruence and suitable dilutions with the sample cakes with a small panel (5 members). These candidates were considered based on literature $[35,39,42,43]$. The odorant candidates were ethyl butanoate, methyl hexanoate, isoamyl acetate, vanillin, maltol, furaneol, $\gamma$-decalactone, ethyl cinnamate, citral and raspberry ketone, all bought from Sigma-Aldrich (Darmstadt, Germany). Additionally, commercial strawberry jam (Saarioinen Oy) and vanilla extract (Dr. Oetker) were included in the odorant screening. The candidate odors were dissolved in propylene glycol in $1 / 30$ to $1 / 150$ dilutions and presented with 3-digit codes. First each panelist described the odors individually and evaluated their congruence with a sweet sponge cake product. After a consensus discussion the most congruent candidate odors were selected for further study.
Based on the screening, six odorants were selected for study 1: isoamyl acetate (1/250 dilution), maltol (1/50 dilution), citral (1/150 dilution), $\gamma$-decalactone (1/80 dilution), strawberry jam (undiluted) and vanilla extract (undiluted but already an aqueous ethanol solution as a retail product). Five to ten microliters of the dilutions (and $3 \mathrm{~g}$ of the jam) were pipetted in $30 \mathrm{ml}$ brown glass vials with caps and provided with the sample cakes.

Maltol (10 g of 1/50 dilution in propylene glycol), strawberry jam (15 $\mathrm{g}$ of undiluted, homogenized jam), and vanilla extract (10 $\mathrm{g}$ of $1 / 10$ dilution in $10 \%$ ethanol) were further used in study 2 . The odorants were pipetted in $50 \mathrm{ml}$ brown glass bottles and delivered to the nasal area of the assessors with the odor display.

\subsection{Sample cakes}

Cake recipes with three varying sugar contents were designed with endosperm rye flour (Fazer Mills, Finland) as the cereal ingredient. Other ingredients included whole egg powder, sugar, baking powder, and rapeseed oil. The sugar contents of the cakes were 25 $\mathrm{g} / 100 \mathrm{~g}$ cake (normal cake), $17 \mathrm{~g} / 100 \mathrm{~g}$ cake (reduced sugar cake), and $12 \mathrm{~g} / 100 \mathrm{~g}$ cake (-50\% sugar cake). The reduced sugar in recipes was replaced by additional endosperm rye flour and water. The aim was to produce samples with higher dietary fiber contents than wheat-based cakes and to retain as similar textures as possible despite the changing sweetness intensity.

The cakes were baked by mixing egg powder with water, followed by sugar addition and whisking. Next rapeseed oil was added to the egg-sugar mixture. Finally, rye flour and baking powder were added. The batters were poured in metal cake pans and the cakes were baked in a rotating rack oven for $45 \mathrm{~min}$ at $175^{\circ} \mathrm{C}$ followed by 10 minutes at $160^{\circ} \mathrm{C}$ (covered by foil). For the evaluation, the crust parts of the cakes (top, bottom and sides) were cut out and the resulting inner crumb was cut to $2 \times 2 \times 2 \mathrm{~cm}$ sample cubes and served monadically along with the paired odor in covered plastic cups. The samples were marked with 3-digit number codes.

\subsection{Odor display}

The odors were produced with a headspace technique based on pushing air through $50 \mathrm{ml}$ bottles (Rixius, Germany) containing odorous material. To achieve a controlled airflow, we developed a custom odor display (shown in Figure 1). A compressor (HBM AS48, Waddinxveen, Netherlands) produced air that was used as the carrier gas. Polytetrafluoroethylene (PTFE) tubing and steel connectors were used between the different components of the display. The air was purified with a cylinder containing activated carbon. The flow of the carrier gas was set to $1.1 \mathrm{~L} / \mathrm{min}$ with a Qflow rotameter (Vögtlin Instruments, Switzerland). The air was then directed to a valve manifold (VX210A08, SMC Corporation, Japan) with eight solenoid valves (VX2A0BZ1S, SMC Corporation, Japan). Solenoid valves have been used earlier for controlling airflow in odor displays [10]. 


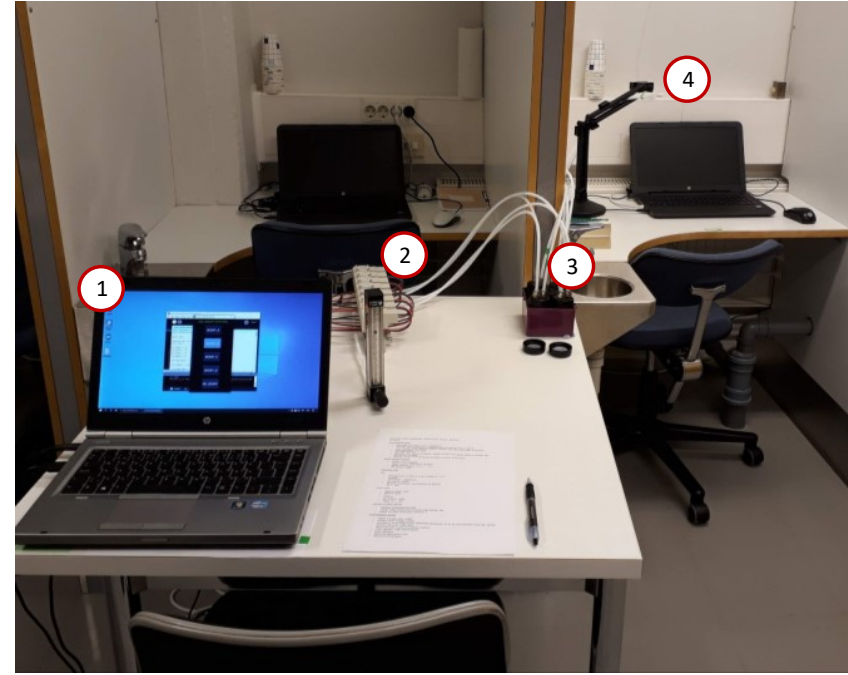

Figure 1. The experimental setup in study 2 consisting of the experimenter's PC (1), solenoid valves (2), odorants in jars (3), and tube outlets (4) for delivering the odors to participants.

Four valves were utilized in the current experiment. Power for each valve was controlled separately with MOSFET transistors connected to an Arduino Mega microcontroller. The Arduino was connected to a laptop PC. The PC was running a graphical interface implemented with Processing (version 3.5.3) which the experimenter used for opening and closing valves. Once the experimenter opened a valve, the air was directed through a bottle. The outlet of the PTFE tube carrying odorous air was positioned close to the participant so that she could perceive the odor while eating the cake sample. Separate tubes and outlets were used for each odor to minimize cross-contamination of odors. The participants were seated so that the tube outlet was approximately $10 \mathrm{~cm}$ away from their nose at the beginning of the evaluation and were monitored to keep this distance throughout the evaluation session.

\subsection{Procedure}

All evaluations were made in the sensory evaluation laboratory (ISO-8589). The laboratory had a $22{ }^{\circ} \mathrm{C}$ ambient temperature and $50 \%$ relative humidity. The experimental procedure followed a typical descriptive profiling approach with panel training and repeated evaluations [14]. The panelists were asked to smell the odorant first, and then eat the sample cake while continuing to smell the odorant. After this, the panelists were asked to evaluate the combined perceived sweetness of the cake-odorant pair and the perceived intensity of the other flavor in the sample pair. Other flavor intensity was defined as the intensity of non-sweetness related odors and flavors in the nasal and oral cavities during chewing of the cake. The evaluation was done with $0-10$ line scales typical to sensory profiling $[14,43]$ where 0 was non-perceivable and 10 was labeled as very intense for the product segment. The panelists could also freely comment on the sample pair after the evaluation. The panelists were instructed to smell and drink water between samples to rinse their palate.

In study 1, the aim was to pre-test the perceived sweetness of different odorants. The odor samples were in odor bottles that the panelists smelled themselves. Study 1 included the $17 \mathrm{~g}$ sugar/100 g cake and $12 \mathrm{~g}$ sugar/100 g cake samples. Blank odor bottles with no added odor were included as hidden controls in the sample design with both cake types. The $17 \%$ sugar content cake with the blank odor bottle pair was available as a reference product with a bound sweetness intensity of 7 . The data was collected in two duplicate sessions in a complete block design with a randomized sample order. The data was collected with Compusense five (Compusense Inc., Guelph, Canada) sensory evaluation software.

In study 2, the aim was to study the effect of odors on perceived sweetness using the odor display. The same sensory attributes and general setup was utilized as in study 1 . However, the odors of the cake-odor pair were delivered with the odor display (Figure 1). For each sample, the assessors smelled the odorant (or clean air) for 10 seconds before tasting the sample cake and proceeding with the intensity evaluation. Additionally, the sample cakes were the $25 \%$ sugar content and $12 \%$ sugar content cakes. Both sample cakes paired with non-odorized air were included as control samples. They were also available to the panelists as references: the $25 \%$ sugar content pair was tied to sweetness intensity 9 , while the $12 \%$ sugar content cake was tied to sweetness intensity 4 . A pause of 45 seconds was forced between the samples to reduce odor carryover effects.

\subsection{Data analysis}

The resulting sensory data of both study 1 and study 2 were analyzed with a two-way mixed model analysis of variance with the samples as a fixed effect and the panelists as a random effect. Tukey's HSD was used as the post hoc test.

\section{RESULTS}

In study 1 , the $12 \mathrm{~g}$ sugar/100 $\mathrm{g}$ cake samples paired with maltol, strawberry jam, and vanilla extract were evaluated to be of approximately the same sweetness as the $17 \mathrm{~g}$ sugar/100 $\mathrm{g}$ cake sample (data not shown). These three compounds were selected thus for study 2. The panel performance was repeatable: the $17 \%$ sugar content cake was evaluated to be close to its bound sweetness intensity when it was presented as a sample. On the other hand, isoamyl acetate had almost zero influence on the perceived sweetness while $\gamma$-decalactone and citral even decreased the perceived sweetness of the $12 \%$ sugar content cake.

In study 2, all three locally delivered odorants augmented the perceived sweetness of the sample-odorant pair (product effect $\mathrm{p}<0.005$ in the two-way ANOVA $[\mathrm{F}(4,36)=35.5])$ compared to the cake with deodorized air only (Figure 2). Along with the rated intensities, most assessors described the odors and flavors in the sample pairs (Table 1). Based on these responses, the assessors identified the odorants well. 

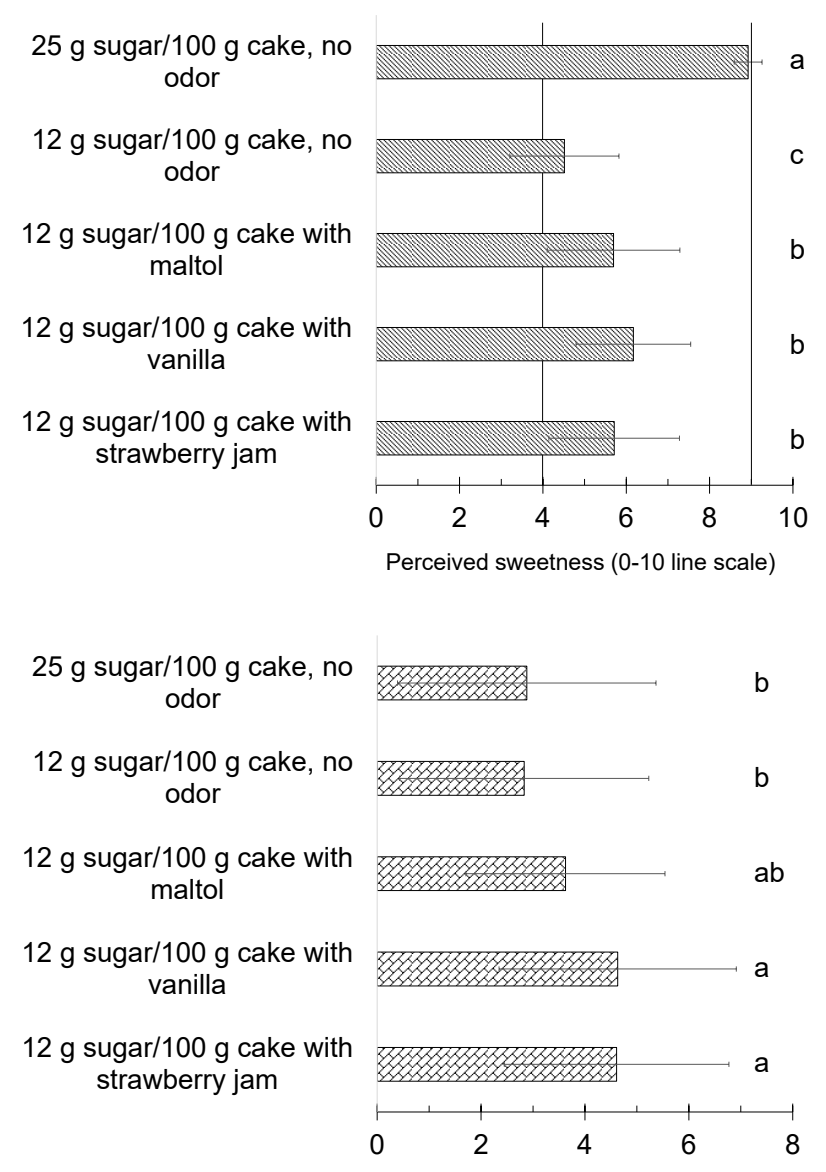

Perceived intensity of other flavor (0-10 line scale)

Figure 2. Results of study 2 . The sweetness and other flavor intensities were evaluated with 0-10 line scales. Sweetness intensities of the $25 \%$ and $12 \%$ sugar content cakes with no odor were bound to 9 and 4 , respectively (vertical lines). The error bars are standard deviations of the 10 assessors and two duplicate measurements. Odor pairs with different letters a-c have statistically significant differences (Tukey's HSD post hoc test).

Table 1. Free descriptions from the panel assessors in Study 2. The descriptions in brackets are less common additions to the typical response.

\begin{tabular}{l|l}
$\begin{array}{l}\text { Odorant in } \\
\text { sample }\end{array}$ & Free comments \\
\hline no odor & plastic (from PTFE tube) \\
\hline maltol & $\begin{array}{l}\text { cotton candy, (cake, } \\
\text { honey) }\end{array}$ \\
\hline vanilla & vanilla (in alcohol) \\
\hline strawberry & (intense) strawberry (jam)
\end{tabular}

The panel repeatability was good as the hidden blank samples were evaluated to be close to their bound reference value sweetness intensities. The evaluation of other flavor intensity was utilized to reduce dumping and the halo effect of sweetness. As expected, the cake sample pairs with no added odorant had the lowest other flavor values. However, maltol interestingly had a trend for a lower other flavor intensity than the other odors. This might be due to the odorant being less familiar in the cake context.

\section{DISCUSSION}

The two studies demonstrated that the perceived sweetness of reduced-sugar cakes can be augmented with locally delivered odorants. This finding with reduced-sugar cakes complements an earlier study [29] with commercial products such as candies and sugar sweetened beverages. The current results suggest that it is possible to augment the sweetness while retaining the lower sugar content. This has many benefits for the consumer because a sweet perception can be achieved with a reduced caloric intake. Using localized odorants circumvents the challenges of low-calorie sweeteners such as the negative public image of aspartame and the laxative effects of sugar alcohols.

In traditional odor-taste crossmodality research, the odorants prepared for sensory evaluations have been mixed in with the tastants as a solution [34,43]. However, in our approach, a localized, but external odor delivery method was employed. This is not an entirely unique approach [29], but a less studied one and similar to earlier VR scenarios [10].

The possible mechanisms of sweetness augmentation with localized odors have been discussed earlier [29]. The mechanisms range from contextual interaction to transferring the sweet quality of the odor to the food matrix. From the viewpoint of HCI research, this means that successful augmentation can be achieved via various mechanisms. The associations of odorants to specific foods also vary as a function of previous experiences, which may also affect the augmentation efficiency. The different mechanisms as well as individual odor associations will be investigated in future research done with consumers rather than trained sensory assessors.

Because we relied on participants' subjective estimations of the perceived sweetness, it is possible that the perceived intensity, familiarity, and pleasantness of the odors were confounding factors in the study. However, this is unlikely because the participants were members of a trained sensory panel who have been trained to analytically deconstruct the sensory experience and to minimize the influence of their subjective preferences.

Even though our findings indicate that the current olfactory display succeeded in augmenting sweetness perception, there are multiple ways to improve the display. For example, we plan to develop an alternative odor delivery method based on a body-mounted system where the odor output is integrated to a necklace $[1,36]$ or an AR headset. This could make the odor delivery more localized and improve the comfort of use. In addition, we will add secondary valves after each odorant jar to ensure that only a single odor is presented at a time. 
The work reported in this paper provides a starting point for our future experiments ( $\mathrm{N}=60-100$ per experiment) that focus on studying how eating experiences can be changed by augmenting food products with olfactory and other sensory cues. The current findings indicate that strawberry jam and vanilla extract are good olfactory cue candidates. In addition, we plan to use also visual cues that change the appearance of food products as suggested by earlier work [17-19,37,38]. The participants will see the visual cues through an AR headset while eating the products. We hypothesize that the number of different sensory cues (such as olfactory and visual augmentation) and their congruence influences the effectiveness of augmentation. The experiments will be carried out with consumers instead of trained sensory panelists, and we also intend to use psycho-physiological measures to complement the more commonly used self-reports. The general aim of our research is to understand whether augmented food products can help in nudging people towards healthier food choices and thus assist in promoting well-being.

\section{ACKNOWLEDGMENTS}

The authors would like to thank Leila Kostamo for her skilled technical assistance. This work was funded by the Academy of Finland (grant numbers 316804 and 316805).

\section{REFERENCES}

[1] Judith Amores and Pattie Maes. 2017. Essence. In Proceedings of the $2017 \mathrm{CHI}$ Conference on Human Factors in Computing Systems - CHI '17, 28-34. https://doi.org/10.1145/3025453.3026004

[2] Malika Auvray and Charles Spence. 2008. The multisensory perception of flavor. Consciousness and Cognition 17, 3: 1016-1031. https://doi.org/10.1016/j.concog.2007.06.005

[3] Felipe Reinoso Carvalho, Kris Steenhaut, Raymond van Ee, Abdellah Touhafi, and Carlos Velasco. 2016. Sound-enhanced gustatory experiences and technology. In Proceedings of the 1st Workshop on Multi-sensorial Approaches to Human-Food Interaction - MHFI '16, https://doi.org/10.1145/3007577.3007580

[4] Jane E. Clark. 1998. Taste and flavour: their importance in food choice and acceptance. Proceedings of the Nutrition Society 57, 4: 639-643. https://doi.org/10.1079/PNS19980093

[5] Rob Comber, Jaz Hee-jeong Choi, Jettie Hoonhout, and Kenton O'Hara. 2014 Designing for human-food interaction: An introduction to the special issue on 'food and interaction design.' International Fournal of Human-Computer Studies 72, 2: 181-184. https://doi.org/10.1016/j.ijhcs.2013.09.001

[6] E.C. Crofton, C. Botinestean, M. Fenelon, and E. Gallagher. 2019. Potential applications for virtual and augmented reality technologies in sensory science. Innovative Food Science \& Emerging Technologies 56, November 2018: 102178. https://doi.org/10.1016/j.ifset.2019.102178

[7] Masaharu Hirose, Karin Iwazaki, Kozue Nojiri, Minato Takeda, Yuta Sugiura, and Masahiko Inami. 2015. Gravitamine Spice: A System that Changes the Perception of Eating through Virtual Weight Sensation. In Proceedings of the 6th Augmented Human International Conference on - AH '15, 33-40. https://doi.org/10.1145/2735711.2735795

[8] Gijs Huisman, Merijn Bruijnes, and Dirk K J Heylen. 2016. A Moving Feast: Effects of Color, Shape and Animation on Taste Associations and Taste Perceptions. Proceedings of the 13th International Conference on Advances in Computer Entertainment Technology: 13:1--13:12. https://doi.org/10.1145/3001773.3001776

[9] Sakiko Ikeno, Ryo Watanabe, Ryuta Okazaki, Taku Hachisu, Michi Sato, and Hiroyuki Kajimoto. 2015. Change in the Amount Poured as a Result of Vibration When Pouring a Liquid. In Lecture Notes in Electrical Engineering. 7-11. https://doi.org/10.1007/978-4-431-55690-9_2

[10] Matthieu Ischer, Naëm Baron, Christophe Mermoud, Isabelle Cayeux, Christelle Porcherot, David Sander, and Sylvain Delplanque. 2014. How incorporation of scents could enhance immersive virtual experiences. Frontiers in Psychology 5, JUL: $1-11$. https://doi.org/10.3389/fpsyg.2014.00736

[11] ISO. 2012. ISO 8586:2012 Sensory analysis - General guidelines for the selection, training and monitoring of selected assessors and expert sensory assessors.
[12] Naoya Koizumi, Hidekazu Tanaka, Yuji Uema, and Masahiko Inami. 2011. Chewing Jockey: Augmented food texture by using sound based on the crossmodal effect. ACM International Conference Proceeding Series. https://doi.org/10.1145/2071423.2071449

[13] Egon P. Köster and Jozina Mojet. 2015. From mood to food and from food to mood: A psychological perspective on the measurement of food-related emotions in consumer research. Food Research International 76, P2: 180-191. https://doi.org/10.1016/j.foodres.2015.04.006

[14] Harry T. Lawless and Hildegarde Heymann. 2010. Descriptive Analysis. In Sensory Evaluation of Food. Springer New York, New York, NY, 227-258. https://doi.org/10.1007/978-1-4419-6488-5

[15] M Lennernas, C Fjellstrom, W Becker, I Giachetti, A Schmitt, A M R de Winter, and M (National Institute of Public Health Kearney Stockholm (Sweden)). 1997. Influences on food choice perceived to be important by nationallyrepresentative samples of adults in the European Union. European fournal of Clinical Nutrition (United Kingdom) v. 51.

[16] Emanuela Maggioni, Robert Cobden, and Marianna Obrist. 2019. OWidgets: A toolkit to enable smell-based experience design. International fournal of Human-Computer Studies 130: 248-260. https://doi.org/10.1016/j.ijhcs.2019.06.014

[17] Takuji Narumi, Yuki Ban, Takashi Kajinami, Tomohiro Tanikawa, and Michitaka Hirose. 2012. Augmented Perception of Satiety: Controlling Food Consumption by Changing Apparent Size of Food with Augmented Reality. In Proceedings of the 2012 ACM annual conference on Human Factors in Computing Systems - CHI '12, 109. https://doi.org/10.1145/2207676.2207693

[18] Takuji Narumi, Takashi Kajinami, Tomohiro Tanikawa, and Michitaka Hirose. 2010. Meta cookie. ACM SIGGRAPH 2010 Emerging Technologies on - SIGGRAPH '10: 1-1. https://doi.org/10.1145/1836821.1836839

[19] Takuji Narumi, Shinya Nishizaka, Takashi Kajinami, Tomohiro Tanikawa, and Michitaka Hirose. 2011. Augmented Reality Flavors: Gustatory Display Based on Edible Marker and Cross-Modal Interaction. In Proceedings of the 2011 annual conference on Human factors in computing systems - CHI '11, 93. https://doi.org/10.1145/1978942.1978957

[20] Takuji Narumi, Shinya Nishizaka, Takashi Kajinami, Tomohiro Tanikawa, and Michitaka Hirose. 2011. Meta Cookie+: An Illusion-Based Gustatory Display. In Virtual and Mixed Reality - New Trends, 260-269.

[21] Masahiro Nishizawa, Wanting Jiang, and Katsunori Okajima. 2016. ProjectiveAR system for customizing the appearance and taste of food. In Proceedings of the 2016 workshop on Multimodal Virtual and Augmented Reality - MVAR '16, 16. https://doi.org/10.1145/3001959.3001966

[22] Saara Pentikäinen, Anne Arvola, Leila Karhunen, and Kyösti Pennanen. 2018 Easy-going, rational, susceptible and struggling eaters: A segmentation study based on eating behaviour tendencies. Appetite 120: 212-221. https://doi.org/10.1016/j.appet.2017.09.001

[23] Saara Pentikäinen, Nesli Sozer, Johanna Närväinen, Kirsi Sipilä, Syed Ariful Alam, Raija Liisa Heiniö, Jussi Paananen, Kaisa Poutanen, and Marjukka Kolehmainen. 2017. Do rye product structure, product perceptions and ora processing modulate satiety? Food Quality and Preference 60, March: 178-187. https://doi.org/10.1016/j.foodqual.2017.04.011

[24] Betina Piqueras-Fiszman and Charles Spence. 2015. Sensory expectations based on product-extrinsic food cues: An interdisciplinary review of the empirical evidence and theoretical accounts. Food Quality and Preference 40, PA: 165-179. https://doi.org/10.1016/j.foodqual.2014.09.013

[25] John Prescott. 2015. Multisensory processes in flavour perception and their influence on food choice. Current Opinion in Food Science 3: 47-52. https://doi.org/10.1016/j.cofs.2015.02.007

[26] Nimesha Ranasinghe, Adrian Cheok, Ryohei Nakatsu, and Ellen Yi-Luen Do. 2013. Simulating the sensation of taste for immersive experiences. In Proceedings of the 2013 ACM international workshop on Immersive media experiences - ImmersiveMe '13, 29-34. https://doi.org/10.1145/2512142.2512148

[27] Nimesha Ranasinghe, Thi Ngoc Tram Nguyen, Yan Liangkun, Lien-Ya Lin, David Tolley, and Ellen Yi-Luen Do. 2017. Vocktail: A Virtual Cocktail for Pairing Digital Taste, Smell, and Color Sensations. In Proceedings of the 2017 $A C M$ on Multimedia Conference - MM '17, 1139-1147. https://doi.org/10.1145/3123266.3123440

[28] Felipe Reinoso Carvalho, Abdellah Touhafi, Kris Steenhaut, Raymond van Ee, and Carlos Velasco. 2017. Using Sound to Enhance Taste Experiences: An Overview. In Bridging People and Sound, 316-330. https://doi.org/10.1007/9783-319-67738-5 19

[29] Paola Risso, Mario Covarrubias Rodriguez, Monica Bordegoni, and Alberto Gallace. 2018. Development and Testing of a Small-Size Olfactometer for the Perception of Food and Beverages in Humans. Frontiers in Digital Humanities 5. https://doi.org/10.3389/fdigh.2018.00007

[30] Peter J. Rogers and Charlotte A. Hardman. 2015. Food reward. What it is and how to measure it. Appetite 90: 1-15. https://doi.org/10.1016/j.appet.2015.02.032

[31] John R. Speakman, David A. Levitsky, David B. Allison, Molly S. Bray, J. M. de Castro, Deborah J. Clegg, John C. Clapham, Abdul G. Dulloo, Laurence Gruer, Sally Haw, Johannes Hebebrand, Marion M. Hetherington, Susanne Higgs, Susan A. Jebb, R. J. F. Loos, Simon Luckman, Amy Luke, Vidya Mohammed-Ali, 
Stephen O'Rahilly, Mark Pereira, Louis Perusse, Tom N. Robinson, Barbara Rolls, Michael E. Symonds, and Margriet S. Westerterp-Plantenga. 2011. Set points, settling points and some alternative models: theoretical options to understand how genes and environments combine to regulate body adiposity. Disease Models \& Mechanisms 4, 6: 733-745. https://doi.org/10.1242/dmm.008698

[32] Charles Spence. 2015. Multisensory Flavor Perception. Cell 161, 1: 24-35. https://doi.org/10.1016/j.cell.2015.03.007

[33] Alina Stelick, Alexandra G. Penano, Alden C. Riak, and Robin Dando. 2018 Dynamic Context Sensory Testing-A Proof of Concept Study Bringing Virtual Reality to the Sensory Booth. Fournal of Food Science 83, 8: 2047-2051. https://doi.org/10.1111/1750-3841.14275

[34] R. J. Stevenson. 1999. Confusing Tastes and Smells: How Odours can Influence the Perception of Sweet and Sour Tastes. Chemical Senses 24, 6: 627-635. https://doi.org/10.1093/chemse/24.6.627

[35] R. J. Stevenson and M. Mahmut. 2010. Differential context effects between sweet tastes and smells. Attention, Perception \& Psychophysics 72, 8: 2304-2313. https://doi.org/10.3758/APP.72.8.2304

[36] R. Tortell, D. P. Luigi, A. Dozois, S. Bouchard, J. F. Morie, and D. Ilan. 2007. The effects of scent and game play experience on memory of a virtual environment. Virtual Reality 11, 1: 61-68. https://doi.org/10.1007/s10055-006-0056-0

[37] Nikita Mae B. Tuanquin. 2017. Immersive virtual eating and conditioned food responses. In Proceedings of the 19th ACM International Conference on Multimodal Interaction - ICMI 2017, 618-622. https://doi.org/10.1145/3136755.3137029
[38] Nikita Mae B Tuanquin, Simon Hoermann, Carl James Petersen, and Robert W Lindeman. 2018. The Effects of Olfactory Stimulation and Active Participation on Food Cravings in Virtual Reality. In 2018 IEEE Conference on Virtual Reality and 3D User Interfaces (VR), 709-710. https://doi.org/10.1109/VR.2018.8446279

[39] D. Valentin, C. Chrea, and D. H. Nguyen. 2006. Taste-odour interactions in sweet taste perception. Optimising Sweet Taste in Foods, March 2006: 66-84 https://doi.org/10.1533/9781845691646.1.66

[40] Carlos Velasco, Felipe Reinoso Carvalho, Olivia Petit, and Anton Nijholt. 2016. A multisensory approach for the design of food and drink enhancing sonic systems. In Proceedings of the 1st Workshop on Multi-sensorial Approaches to Human-Food Interaction - MHFI '16, 1-7. https://doi.org/10.1145/3007577.3007578

[41] Carlos Velasco, Marianna Obrist, Olivia Petit, and Charles Spence. 2018 Multisensory technology for flavor augmentation: A mini review. Frontiers in Psychology 9, JAN: 1-6. https://doi.org/10.3389/fpsyg.2018.00026

[42] Gloria Wang, Alyssa J. Bakke, John E. Hayes, and Helene Hopfer. 2019 Demonstrating cross-modal enhancement in a real food with a modified ABX test. Food Quality and Preference 77, January: 206-213. https://doi.org/10.1016/j.foodqual.2019.05.007

[43] Gloria Wang, John Hayes, Gregory Ziegler, Robert Roberts, and Helene Hopfer 2018. Dose-Response Relationships for Vanilla Flavor and Sucrose in Skim Milk Evidence of Synergy. Beverages 4, 4: 73. https://doi.org/10.3390/beverages4040073 